“ (C) 2018 IEEE. Personal use of this material is permitted. Permission from IEEE must be obtained for all other uses, in any current or future media, including

reprinting/republishing this material for advertising or promotional purposes, creating new collective works, for resale or redistribution to servers or lists, or reuse of any copyrighted component of this work in other works." 


\title{
Dual-Linearly-Polarized, Electrically Small, Low-Profile, Broadside Radiating, Huygens Dipole Antenna
}

\author{
Ming-Chun Tang, Senior Member, IEEE, Zhentian Wu, Ting Shi, Student Member, IEEE, Hao Zeng, \\ Wei Lin, Member, IEEE, and Richard W. Ziolkowski, Fellow, IEEE
}

\begin{abstract}
A dual-linearly-polarized, electrically small, low-profile, broadside radiating Huygens dipole antenna is presented that is an advanced combination of electric and magnetic near-field resonant parasitic (NFRP) elements. Its prototype was fabricated and tested. The measured results are in good agreement with their simulated values. At $1.515 \mathrm{GHz}$ the prototype is electrically small $(k a=0.904)$ and low profile $\left(0.0483 \lambda_{0}\right)$. It exhibits high port isolation and a large front-to-back ratio (FTBR). The isolation between its two ports is demonstrated to be over $25.8 \mathrm{~dB}$ within its -10-dB fractional impedance bandwidth (FBW), $0.46 \%$. When port 1 (port 2) is excited, the peak realized gain is $2.03 \mathrm{dBi}(2.15 \mathrm{dBi})$ strictly along the broadside direction with a $12.4 \mathrm{~dB}(12.1 \mathrm{~dB})$ FTBR.
\end{abstract}

Index Terms-Dual polarization, broadside radiation, directivity, electrically small antennas, Huygens dipole antennas, near-field resonant parasitic elements.

\section{INTRODUCTION}

$\mathrm{W}$ ith the intense drive of wireless communication systems towards compactness and integration, electrically small antennas (ESAs) have attracted considerable attention in recent years. However, their directional radiation performance is generally constrained to be that of an electric or magnetic dipole antenna [1]. Consequently, their peak realized gains and

Manuscript received on Sep. 23, 2017; revised on Mar. 21, 2018; and accepted on May 20

This work was supported in part by the National Natural Science Foundation of China contract numbers 61471072 and 61701052, in part by the Funding of the Innovative Leading Talents in Science and Technology of Chongqing contract number CSTCCXLJRC201705, in part by Innovation Funds from China Academy of Space Technology contract number 201707162, in part by the Funding of the leading research talent cultivation plan of Chongqing University contract number cqu2017hbrc1A08, and in part by Funding of the Young Backbone Teachers in Colleges and Universities of Chongqing contract number 0307001104102, and in part by the Australian Research Council grant number DP160102219.

M. -C. Tang, Z. Wu, T. Shi and H. Zeng are with the Key Laboratory of Dependable Service Computing in Cyber Physical Society Ministry of Education, College of Communication Engineering, Chongqing University and also with the Chongqing Engineering Laboratory of High Performance Integrated Circuits, College of Communication Engineering, Chongqing University, Chongqing 400044, China(E-mail: tangmingchun@cqu.edu.cn);

W. Lin is with the University of Technology Sydney, Global Big Data Technologies Centre, Ultimo NSW 2007, Australia;

R. W. Ziolkowski is with the University of Technology Sydney, Global Big Data Technologies Centre, Ultimo NSW 2007, Australia, and the Department of Electrical and Computer Engineering, University of Arizona, Tucson, AZ 85721, USA (E-mail: Richard.Ziolkowski@uts.edu.au). front-to-back ratios (FTBRs) are usually low. In order to empower better directivity for a variety of wireless applications, e.g., long-distance/point-to-point wireless communications [2-3], radio-frequency identification devices (RFIDs) [4-6], and wireless power transfer [7-10], many different approaches have been reported. These include loading the radiating elements with electromagnetic band gap (EBG) structures [11], adding slot structures[12], and employing additional small radiating elements[13-14], only to name a few. In comparison, the method of combining pairs of electric and magnetic dipoles to realize Huygens dipole ESAs commands an inherent advantage. Directivity enhancement $(\sim 3 \mathrm{~dB})$ is achieved without significantly increasing the overall size and profile of the antenna system. Existing Huygens source ESAs can be classified into the two usual polarization categories: linearly polarized (LP) designs [15-21] and circularly polarized (CP) designs [21-23]. However, to the best of our knowledge, all of the reported systems radiate a single polarization state.

Dual-polarized antennas are well-known to have significant advantages over their single-polarized counterparts in many practical engineering applications. They are equivalent to a combination of two different polarized antennas. Thus, they reduce the total number of units in a system and, hence, its cost, weight, and footprint while still maintaining, if not increasing, its communications capabilities and qualities.

High port isolation between each element in a dual-polarized system is an indispensable feature. Numerous effective structures have been examined to accomplish it. Examples include aperture-coupled feed networks [24-26], meandering or cross- probes [27-28], coupled feed networks [29-30], hybrid feed networks of apertures and probes [31], and even-odd mode feed structures [32]. Similarly, several dual-LP magneto-electric dipoles have been reported that employ other technologies. For instance, two separate substrate integrated waveguides (SIWs) and aperture-coupled feed networks were utilized to achieve dual-polarized operations in [33]. The dual-polarization performance characteristics in [34] were accomplished using two orthogonal $\Gamma$-probe coupled feed networks. However, since very large ground planes were required to construct those feed networks [33, 34], their electrical sizes are inescapably quite large, i.e., $k a>3.5$, where $k=2 \pi / \lambda_{0}$ and $a$ is the radius of the smallest sphere that completely encloses the entire antenna system at the 
operational free-space wavelength $\lambda_{0}$. Consequently, their applications in compact wireless communication systems are very limited.

In this paper, an electrically small, low-profile, Huygens dipole antenna with dual-LP radiation performance characteristics is reported. It is based on an advanced combination of magnetic, capacitively loaded loop (CLL), and electric, Egyptian axe dipole (EAD), near-field resonant parasitic (NFRP) elements and the introduction of a compact, balanced feed network specifically tailored to accommodate these NFRP elements. This architecture leads to the large port isolation while maintaining the electrically small size and low profile. The dual-LP antenna design is introduced in Section II. Next, in Section III, the fabrication and assembly of an optimized prototype is described. The measured results are shown to be in agreement with their simulated values. They demonstrate that the prototype is impedance matched to its $50-\Omega$ source at $1.515 \mathrm{GHz}$ and is electrically small $(k a<1)$, low profile, and exhibits high port isolation within its $-10-\mathrm{dB}$ impedance bandwidth. When either port is excited, broadside radiation is attained along with a corresponding large FTBR value $(>10 \mathrm{~dB})$. The operational mechanisms are investigated in detail in Section IV, including the isolation performance, the antenna loss, and the broadside radiation mechanism. Finally, some conclusions are drawn in Section V.

All of the numerical simulations and their optimizations reported herein were performed using the frequency domain, finite-element based ANSYS/ANSOFT High Frequency Structure Simulator (HFSS), version 13. The simulation models employed the known, real properties of the dielectrics and conductors. An operational frequency near $1.5 \mathrm{GHz}$ was selected for the designs to simplify the fabrication of the dual-LP prototype and to match the available measurement equipment. We note that this frequency of operation can be readily shifted to match nearby bands that have narrow-band applications, e.g., radio-frequency identification devices (around $980 \mathrm{MHz}$ ), wireless power transfer devices (around 2.4 $\mathrm{GHz}$ ), and MIMO communication systems (around $3.8 \mathrm{GHz}$ ).

\section{DUAL-LP HUYGENS DiPOLE ESA DESIGN}

The geometric structure of the dual-LP Huygens dipole ESA is shown in Fig. 1 and the corresponding optimized design parameters are given in Table I. As shown in Figs. 1 (a) and (b), the dual-LP Huygens dipole ESA consists of five substrate layers into which the conductors are integrated. They are labeled as Layer_1-Layer_5. All of these copper cladded substrates are Rogers 4350B laminate with a $0.017 \mathrm{~mm}$ copper thickness, relative dielectric constant $\varepsilon_{r}=3.48$ and loss tangent $\tan \delta=0.0037$. All these substrates have the same radius $(\mathrm{R} 1=$ $28.5 \mathrm{~mm}$ ), but have different thicknesses ( $\mathrm{h} 1=\mathrm{h} 2=\mathrm{h} 4=0.254$ $\mathrm{mm}, \mathrm{h} 3=1.508 \mathrm{~mm}, \mathrm{~h} 5=0.508 \mathrm{~mm}$ ).

The magnetic element consists of two orthogonally-oriented CLLs. One is oriented along the $x$-axis, the other along the $y$-axis. The horizontal portions of these magnetic elements are placed on the upper surfaces of Layer_1 and Layer_3. They are connected by copper cylindrical columns passing from Layer_1 to Layer_3. The electric element consists of two Egyptian axe dipoles (EADs), one oriented along the $x$-axis, the other along the $y$-axis. Both are placed on the upper surface of Layer_2. These dual CLLs and EADs operate as NFRP elements. They are excited by the driven dipole strips, which are located on Layer_4 and Layer_5.

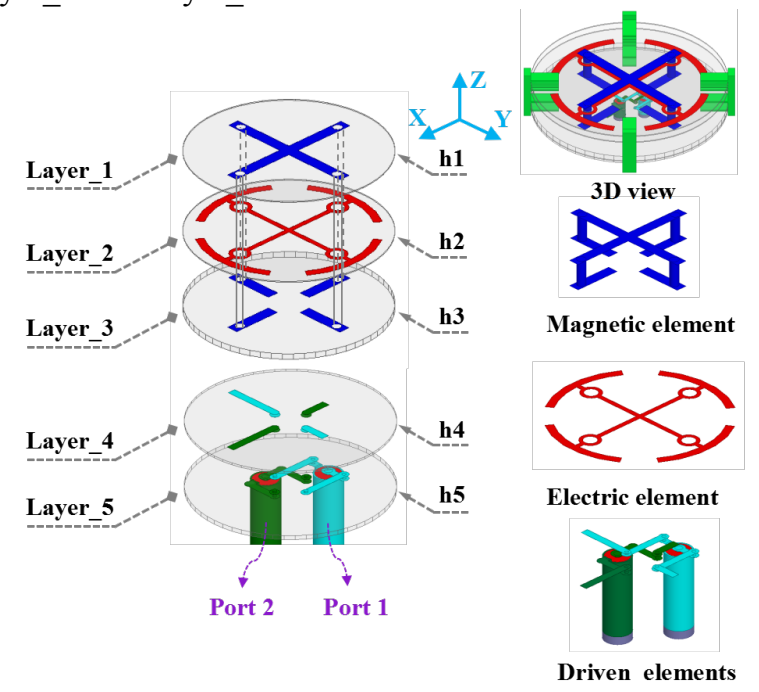

(a)

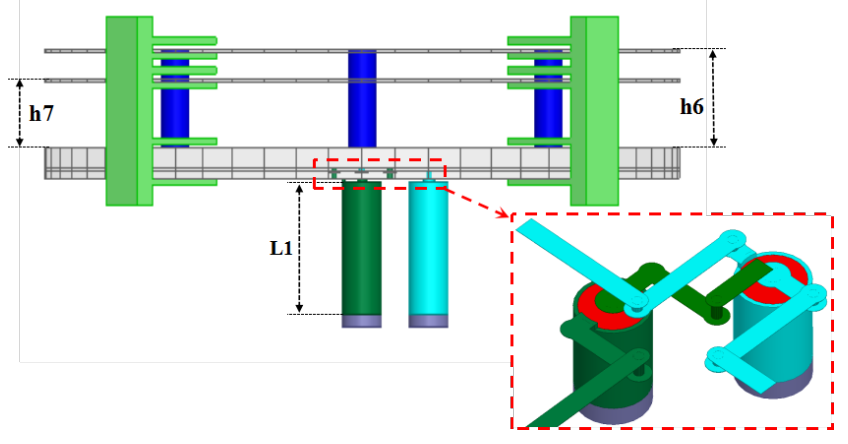

(b)

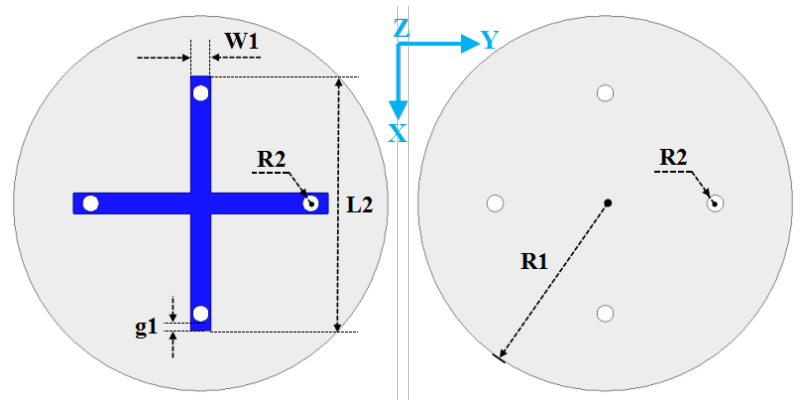

(c)
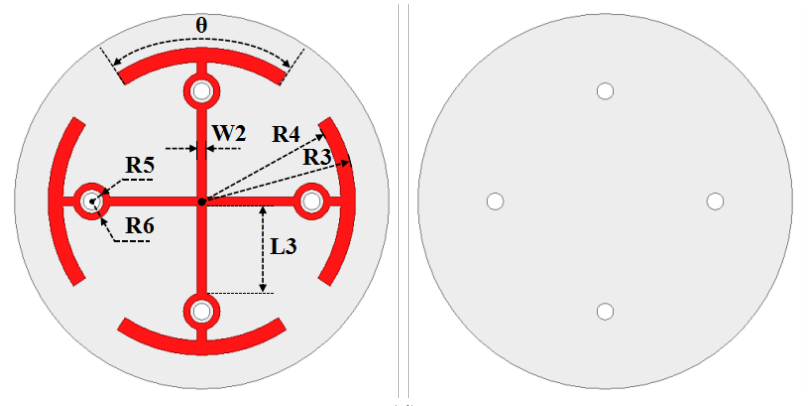

(d) 

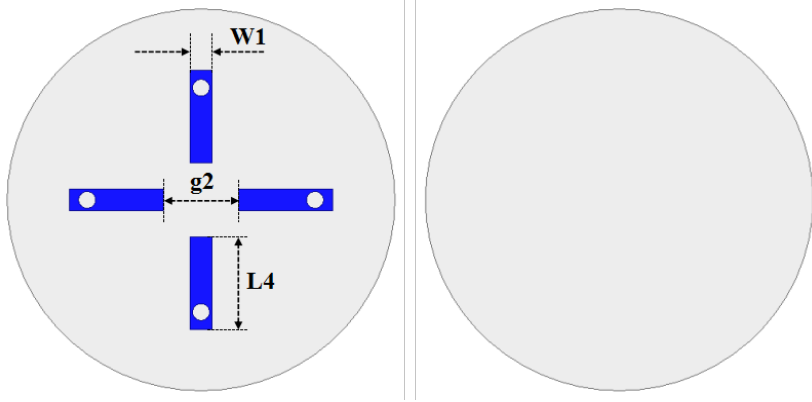

(e)
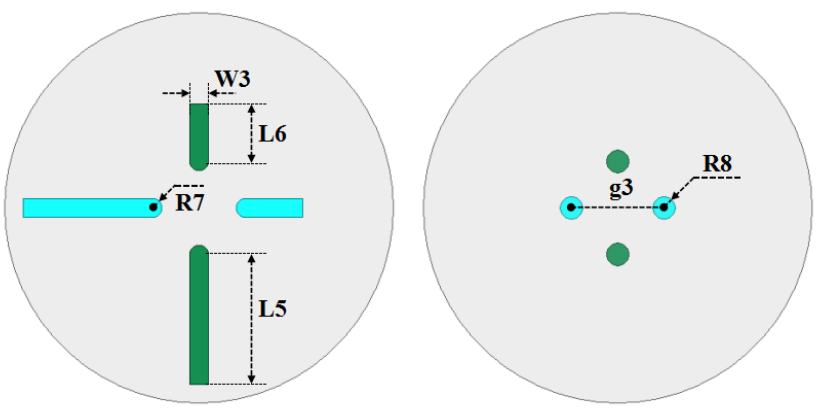

(f)
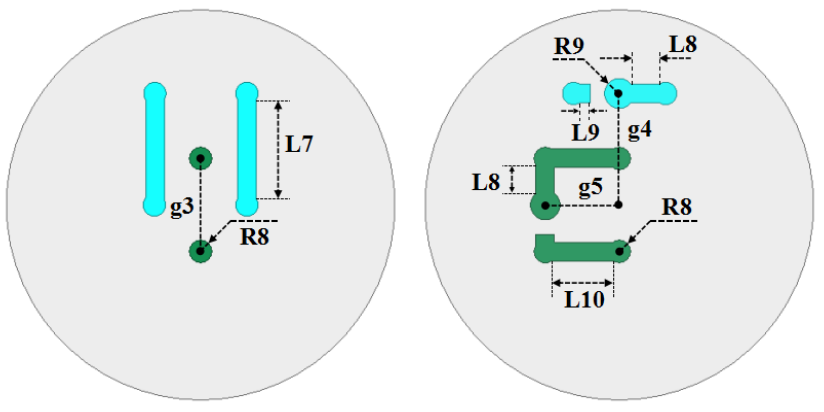

(g)

Fig.1 Geometry of the dual-LP Huygens dipole ESA. (a) 3-D isometric view. (b) Side view. Upper and lower surfaces of (c) Layer_1, (d) Layer_2, (e) Layer_3, (f) Layer_4, and (g) Layer_5.

TABLE I. The OPTIMIZED DUAL-LP HuYGENS DiPOLE ESA PARAMETERS (IN MM)

\begin{tabular}{|c|c|c|c|c|}
\hline$h 1=0.254$ & $h 3=1.508$ & $h 5=0.508$ & $h 6=7.3$ & $h 7=5.1$ \\
\hline$L 2=38.8$ & $L 3=13.3$ & $L 4=13.9$ & $L 5=7.0$ & $L 6=3.1$ \\
\hline$L 7=5.3$ & $L 8=1.54$ & $L 9=0.57$ & $L 10=3.37$ & $W 1=3.1$ \\
\hline$W 2=1.4$ & $W 3=1.0$ & $g 1=1.4$ & $g 2=11.0$ & $g 3=5.0$ \\
\hline$g 4=6.0$ & $g 5=4.0$ & $R 1=28.5$ & $R 2=1.25$ & $R 3=23.4$ \\
\hline$R 4=21.2$ & $R 5=1.8$ & $R 6=2.8$ & $R 7=0.5$ & $R 8=0.6$ \\
\hline$R 9=0.8$ & $\Theta=66.8^{\circ}$ & \multicolumn{3}{|c|}{ Null } \\
\hline
\end{tabular}

The operating mechanisms of the CLL and EAD NFRP elements, and the design guidelines of the single-polarized Huygens dipole ESAs realized with them have been extensively described previously $[18,21]$. In this dual-LP design, the CLL and EAD elements located along the $y$-axis are both excited by port 1; they generate a LP Huygens radiation pattern along the $y$-axis. Likewise, when excited by port 2, the CLL and EAD elements along the $x$-axis generate the second LP Huygens radiation pattern along it. The related port isolation principles will be presented in Section IV. A.
In more detail, the upper and lower surfaces of each substrate are illustrated in Fig. 1 (c)-(g). Fig. 1 (c) shows that the magnetic element is cross-shaped, each copper strip being L $2 \times \mathrm{W} 1$ in size, and lies on the upper surface of Layer_1. The two orthogonal EAD elements have the same size and are located on the upper side of Layer_2, as shown in Fig. 1 (d). The four rectangular copper strips shown in Fig. 1 (e) are each L4 $\times$ W1 in size and are symmetrically located with respect to the center of the disk. The gaps, g2, between them along the $x$ and $y$-axes are the same size. They are located on the upper surface of Layer_3 and act as the lower face of the magnetic element. The cross-shaped element on Layer_1 and the four copper strips on Layer_3 are connected by four copper cylindrical columns, each with height $\mathrm{h} 6=7.3 \mathrm{~mm}$ and radius $\mathrm{R} 2=1.25 \mathrm{~mm}$. These four copper cylindrical columns pass completely through Layer_2, as shown in Fig. 1 (b). They pass through the four copper rings located on Layer_2 shown in Fig. 1 (d). They have an inner radius equal to $3.3 \mathrm{~mm}$ and are introduced on the electric element to avoid it being shorted to the magnetic element.

The driven elements are shown in Figs. 1 (f) and (g). They are designed to lie on both of the surfaces of both Layer 4 and Layer_5. There are two pairs of copper strips with the same size lying on the upper surface of Layer_4. They serve as the feedlines from port 1 and port 2 . One pair is placed along the $y$-axis, the other pair along the $x$-axis. Port 1 (port 2) is placed a distance g4 (g5) away from the center of the substrate along the $x$-axis (y-axis) on the lower surface of Layer 5. The inner and outer conductors of the $50-\Omega$ coaxial cable representing port 1 are connected directly to the two short (blue) copper strips on the lower surface of Layer_5 along the $y$-axis. The two same-sized copper strips on the upper surface of Layer_5 are connected to them by two vertical vias that are $0.25 \mathrm{~mm}$ in radius. Similarly, the inner and outer conductors of the second $50-\Omega$ coaxial cable representing port 2 are connected directly to the two longer (green) copper strips on the lower surface of Layer 5 also oriented along the $y$-axis. The two copper pads (green) on the upper surface of Layer_5 are connected to them by two vertical vias that are $0.25 \mathrm{~mm}$ in radius. The copper strips on the upper surface of Layer_4 and four copper pads on the lower surface of Layer_4 are also connected by vias whose radii are $0.25 \mathrm{~mm}$. In order to guarantee alignment of the four vias penetrating through Layer_4 and Layer_5, four pairs of copper pads, each having the radius $\mathrm{R} 8=0.6 \mathrm{~mm}$, are etched as two pairs on the lower surface of Layer_4 and two pairs on the upper surface of Layer_5. With this two-layer feed structure, the feedlines of both ports exhibit a $90^{\circ}$ rotational symmetry. Furthermore, in the assembly process, Layer_3, Layer_4 and Layer 5 are adhered together using a silicone rubber adhesive. Four $3 \mathrm{D}$ printed polyamide brackets are used to maintain the relative vertical distance between Layer_1 and Layer_2 to ensure the mechanical stability of the assembled antenna.

\section{Simulated AND MEASURED Results}

The dual-LP Huygens dipole ESA in Fig. 1 was fabricated, assembled and measured. A photo of all of its component parts before assembly is presented in Fig. 2 (a). The assembled 
antenna mounted on two sleeve baluns, one for each port, is shown in Fig. 2 (b). The sleeve baluns are introduced simply to mitigate any cable effects associated with the measurement process. The S-parameters of the dual-LP Huygens dipole ESA were measured with an Agilent E8361A PNA vector network analyzer (VNA) whose potential measurement scale was 0.01-67.0 GHz. Its far-field radiation performance characteristics were measured in an anechoic chamber at UESTC, Chengdu, China which utilizes an Agilent N5230A PNA-L VNA and a SATIMO passive measurement system. The antenna under test (AUT) in this chamber is shown in Fig. 2 (c).

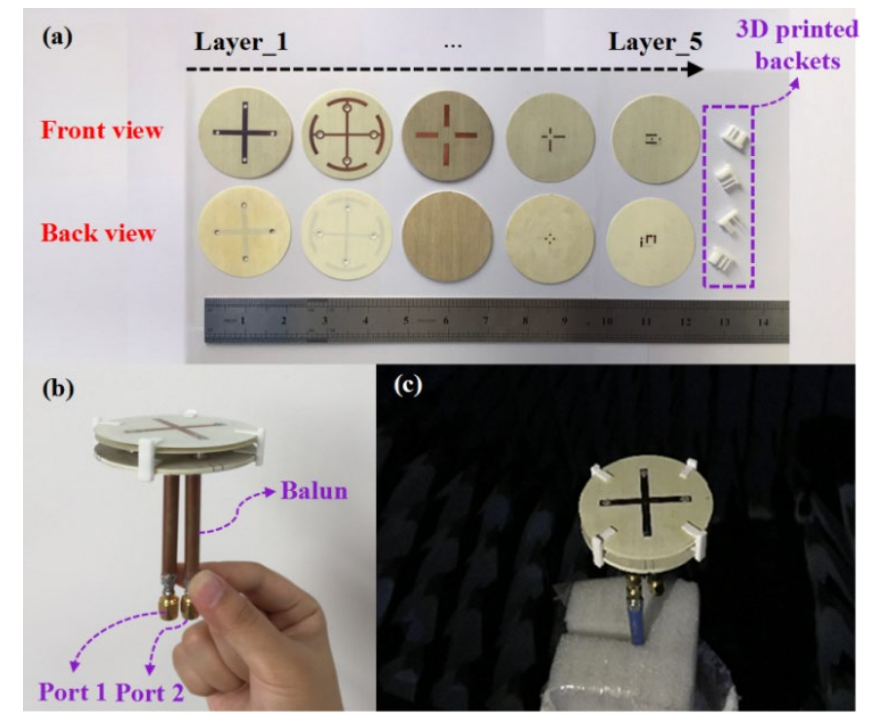

Fig. 2 Fabricated prototype of the dual-LP Huygens dipole ESA. (a) Front and back views of each layer before assembly. (b) Side view of the assembled antenna mounted on two sleeve baluns, one for each port. (c) 3-D isometric view of the antenna under test (AUT) in the anechoic measurement chamber.

As demonstrated with our simulation studies, the length of the coaxial line has a non-negligible impact on both the impedance and radiation performance of the dual-LP ESA. These results are shown in Fig. 3 (a). The reason is that the direct connections between the balanced dipoles and the unbalanced coaxial cables lead to unwanted surface currents that leak onto the outer conductor of the coaxial cables [35-37]. These undesired surface currents degrade the impedance matching and radiation performance significantly. Therefore, a $49 \mathrm{~mm}$-long (nearly one-quarter wavelength) sleeve balun was added to each port as shown in Fig. 2 (b) to choke-off those unwanted surface currents. In particular, each balun was connected to the outer conductor of the coaxial cable feeding each port. The terminal end of each balun was arranged to be very close to the lower surface of Layer_5.

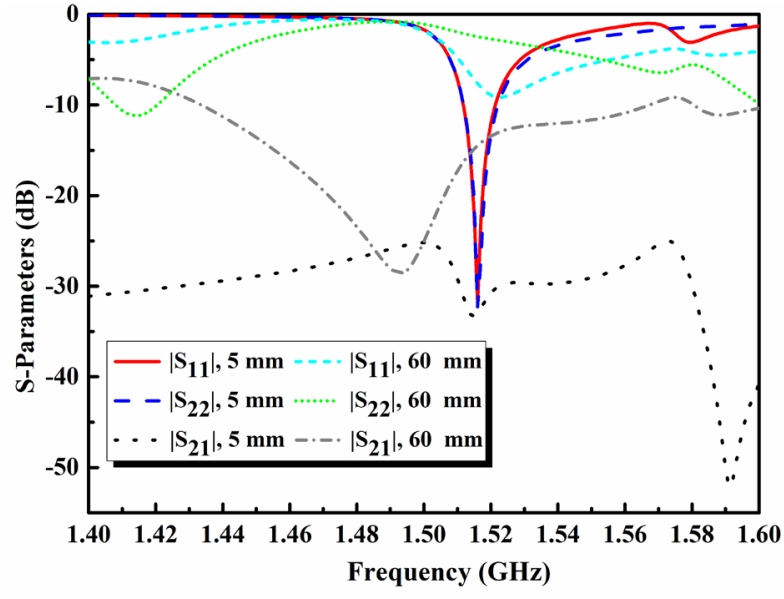

(a)

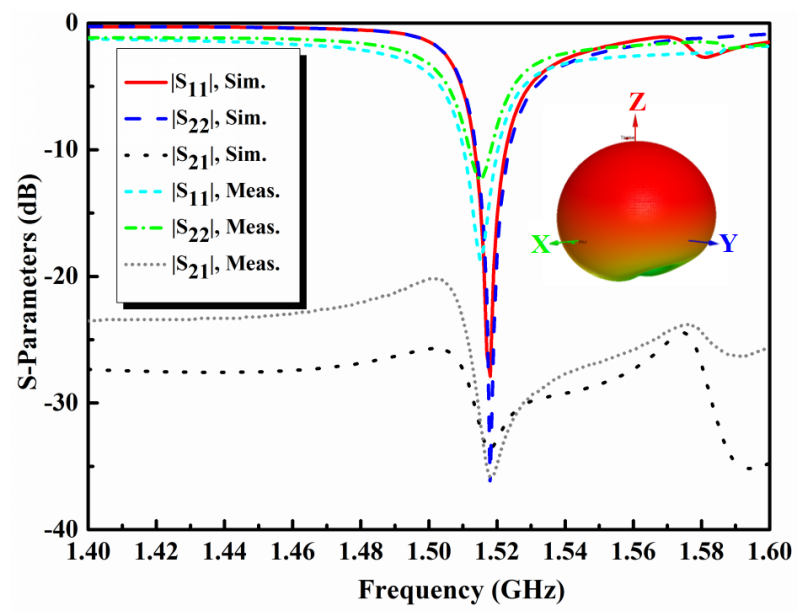

(b)

Fig. 3 Simulated and measured S-parameters of the dual-LP Huygens dipole ESA. (a) Simulated S-parameters when the coaxial cable length $\mathrm{L} 1=5 \mathrm{~mm}$ and $60 \mathrm{~mm}$ without the baluns. (b) Simulated and measured S-parameters with the baluns, together with its simulated 3-D directivity pattern.

The simulated (taking into account the presence of the baluns) and measured results of the dual-LP Huygens dipole ESA are shown in Fig. 3 (b). The simulated (measured) results demonstrate that the resonance frequencies of both states of this antenna are centered at $1.518 \mathrm{GHz}(1.515 \mathrm{GHz})$ with $\left|\mathrm{S}_{11}\right|_{\min }=$ $-27.8 \mathrm{~dB}(-17.8 \mathrm{~dB})$ and $\left|\mathrm{S}_{22}\right|_{\min }=-36.5 \mathrm{~dB}(-12.4 \mathrm{~dB})$. The corresponding impedance bandwidth was $9 \mathrm{MHz}(7 \mathrm{MHz})$. The isolation between the two ports is better than $30.6 \mathrm{~dB}(25.8 \mathrm{~dB})$ within the operational bandwidth. Thus, the simulated (measured) total height and electrical size of this dual-LP ESA at the resonance frequency $\left(\lambda_{0, \text { sim }}=197.62 \mathrm{~mm}, \lambda_{0, \text { meas }}=198.01\right.$ $\mathrm{mm})$ were $0.0484 \lambda_{0}\left(0.0483 \lambda_{0}\right)$ and $k a=0.906(0.904)$, respectively. These measured results are in good agreement with their simulated values; they confirm that the measured dual-LP Huygens dipole ESA is low-profile and electrically small. 
TABLE II

COMParison Of The Dual-LP Huygens Dipole Antenna With Related ANTENNAS Reported In The Literature

\begin{tabular}{|c|c|c|c|c|c|c|c|c|}
\hline Category & $\mathrm{ka}$ & $\begin{array}{c}\text { FBW } \\
(\%)\end{array}$ & $\begin{array}{c}\text { Peak } \\
\text { Realized } \\
\text { Gain } \\
(\mathrm{dBi})\end{array}$ & $\begin{array}{c}\text { FTBR } \\
(\mathrm{dB})\end{array}$ & $\begin{array}{c}\text { Isolation } \\
(\mathrm{dB})\end{array}$ & $\begin{array}{c}\text { Transverse Electrical } \\
\text { Size }\end{array}$ & Height & $\begin{array}{c}\text { Ground } \\
\text { Independent }\end{array}$ \\
\hline$[28]$ & 7.990 & 57.5 & 8.8 & 25 & $>31$ & $1.1 \lambda_{0} \times 2.29 \lambda_{0}$ & $0.26 \lambda_{0}$ & no \\
\hline$[34]$ & 3.546 & 48 & 9.3 & 29 & $>30$ & $0.8 \lambda_{0} \times 0.8 \lambda_{0}$ & $0.26 \lambda_{0}$ & no \\
\hline$[38]$ & 4.655 & 56 & 8.9 & - & $>30$ & $1.04 \lambda_{0} \times 1.04 \lambda_{0}$ & $0.34 \lambda_{0}$ & no \\
\hline$[39]$ & 5.695 & 65.9 & 9.5 & - & $>36$ & $1.28 \lambda_{0} \times 1.28 \lambda_{0}$ & $0.23 \lambda_{0}$ & no \\
\hline$[40]$ & 5.775 & 68 & 9.6 & 15 & $>36$ & $1.3 \lambda_{0} \times 1.3 \lambda_{0}$ & $0.24 \lambda_{0}$ & no \\
\hline$[41]$ & 5.110 & 52 & 8.6 & 15 & $>35$ & $1.15 \lambda_{0} \times 1.15 \lambda_{0}$ & $0.25 \lambda_{0}$ & no \\
\hline Rep. Ant. & 0.904 & 0.46 & 2.15 & 12 & $>25.8$ & $0.287 \lambda_{0} \times 0.287 \lambda_{0}$ & $0.048 \lambda_{0}$ & yes \\
\hline
\end{tabular}

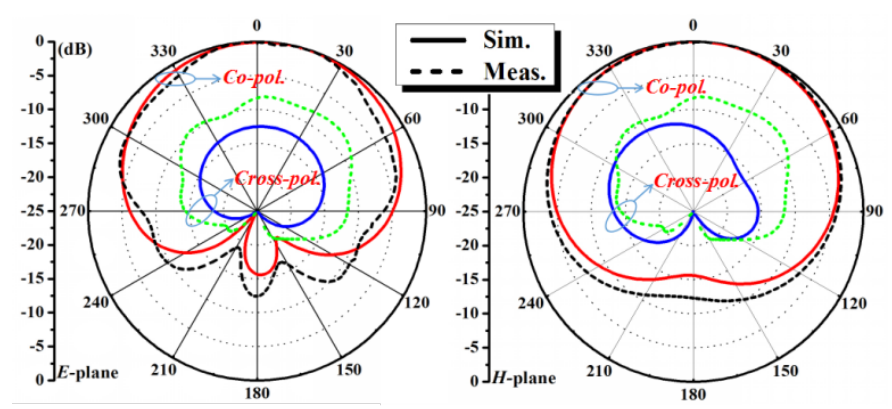

(a)

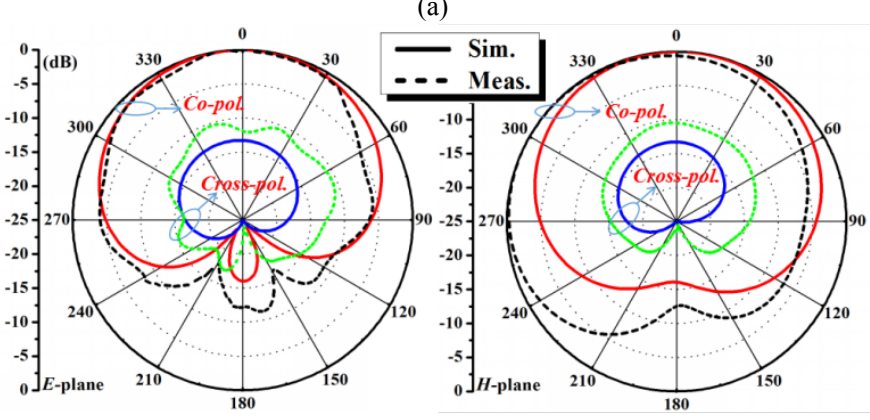

(b)

Fig. 4 Simulated and measured normalized realized gain patterns for each port in the $E$ - and $H$ - planes of the dual-LP Huygens dipole ESA at its resonance frequency ( $1.518 \mathrm{GHz}$ in simulation, $1.515 \mathrm{GHz}$ in experiment). (a) Only port 1 is excited. (b) Only port 2 is excited.

The simulated and measured realized gain patterns of the dual-LP Huygens dipole ESA are shown in Fig. 4. It is clear that good broadside radiation performance was obtained. The main-beam direction for each polarization state is oriented along the $+z$-axis. Specifically, when port 1 was excited, the simulated (measured) peak realized gain value was $2.2 \mathrm{dBi}$ $(2.03 \mathrm{dBi})$ and the FTBR value was $15.6 \mathrm{~dB}(12.4 \mathrm{~dB})$ at 1.518 $\mathrm{GHz}(1.515 \mathrm{GHz})$. The simulated radiation efficiency (RE) value corresponding to the peak realized gain value was $61.3 \%$. The simulated (measured) half-power beamwidth (coverage) was from $-60.8^{\circ}$ to $75.6^{\circ}\left(-68^{\circ}\right.$ to $\left.56^{\circ}\right)$ in the $E$-plane (zOy-plane) and from $-73.9^{\circ}$ to $75.9^{\circ}\left(-78^{\circ}\right.$ to $\left.80^{\circ}\right)$ in the $H$-plane (z0x-plane). The simulated (measured) cross-polarization discrimination (XPD) values in the broadside direction were $12.6 \mathrm{~dB}(8.3 \mathrm{~dB})$ in both the $E$ - and $H$-planes. In contrast, when port 2 was excited, the simulated (measured) peak realized gain value was $2.5 \mathrm{dBi}(2.15 \mathrm{dBi})$ and the FTBR value was $16 \mathrm{~dB}(12.1 \mathrm{~dB})$ at $1.518 \mathrm{GHz}(1.515$ $\mathrm{GHz}$ ). The simulated radiation efficiency (RE) value corresponding to the peak realized gain value was $64 \%$. The simulated (measured) half-power beamwidth (coverage) was from $-73.7^{\circ}$ to $66.6^{\circ}\left(-68^{\circ}\right.$ to $\left.46^{\circ}\right)$ in the $E$-plane ( $z O x$-plane) and from $-70.8^{\circ}$ to $74.4^{\circ}\left(-134^{\circ}\right.$ to $\left.50^{\circ}\right)$ in the $H$-plane ( $z 0 y$-plane). The simulated (measured) XPD values in the broadside direction were $13.7 \mathrm{~dB}(10.5 \mathrm{~dB})$ in both the $E$ - and $H$-planes.

The measured results witness slight differences from their simulated values as observed in Figs. 3 and 4. The main reasons for them are ascribed to the small dimensional errors generated during the fabrication and assembly stages. They include the following. First, the assembly errors associated with the use of the silicone rubber adhesive and the length of the four metal columns impact the relative distances between and parallel orientation of the five substrate layers. Deviations from the optimized design cause the differences between the measured and simulated S-parameter values, especially the impedance matching levels, i.e., $\left|S_{11}\right|_{\text {min }}$ and $\left|S_{22}\right|_{\text {min }}$. Second, the two baluns actually do not completely remove all of the surface currents on the outer conductor of the coaxial lines in the experiment. This leakage also arises from the fabrication and assembly errors associated with the baluns themselves. These errors cause the ripples which appear in the E-plane patterns, and also lead to the measured XPD levels in both states being slightly lower than their simulated values. Third, the larger $H$-plane errors are associated with measurement system issues. The lower FTBR values and a slight tilt of the patterns in the $H$-planes are generated in both states as a consequence.

As was initially emphasized, the goal of our design was to achieve an electrically small dual-LP directional antenna having a low profile. To emphasize the efficacy of our antenna, a comparison of it and several recently reported dual-LP antennas is given in Table II. In order to make this comparison fair and comprehensive, the electrical sizes ( $k a$, where $a$ includes the entire (ground plane and all other elements) antenna structure), fractional bandwidths, peak realized gain values, FTBR values, isolation levels, height and ground-independent performance characteristics are listed. Note that the electrical size corresponds to the center frequency of the operational band defined by its $-10 \mathrm{~dB}$ impedance bandwidth. Clearly, while Table II highlights the good isolation achieved with our design, the listed values also demonstrate 
that it has an electrically small size, low-profile, and high FTBR value without any ground plane. We note that the smaller bandwidth of our design is expected because of its electrically smaller size. On the other hand, its compactness is very attractive for the many existing and potential narrowband applications.

\section{INVESTIGATIONS OF THE OPERATIONAL MECHANISMS}

\section{A. Discussion of the Isolation Performance}

In order to explain the isolation mechanism of the dual-LP Huygens dipole ESA in a comprehensive manner, the surface current distributions on the main copper surfaces of the dual-LP ESA were simulated. They are presented at different times: 0, $\mathrm{T} / 4, \mathrm{~T} / 2$ and $3 \mathrm{~T} / 4$, corresponding to the period $\mathrm{T}$ associated with the resonance frequency, e.g., at $1.518 \mathrm{GHz}$. Given the nearly identical performance of the antenna in both of its states, only the surface current behavior arising from the excitation of port 1 is reported to explain its operation.

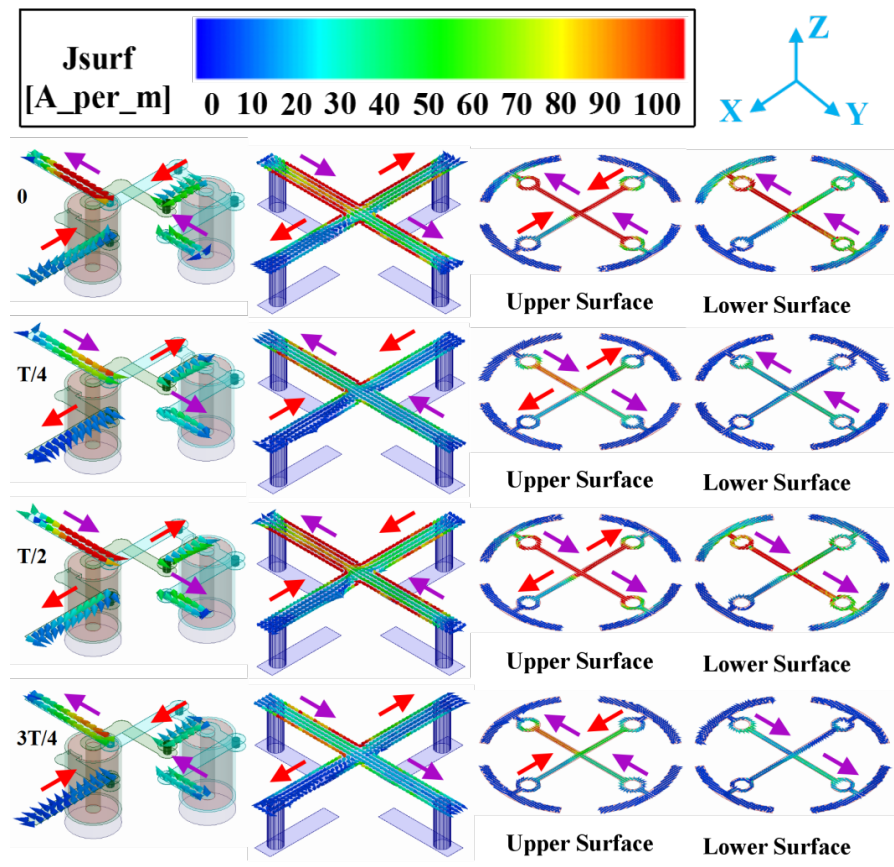

Fig. 5 Surface current distributions on the cross-shaped copper strips of the CLL and EAD NFRP elements and the feedlines of the dual-LP Huygens dipole ESA at several snapshots in time during one period of the resonance frequency. (The purple arrows represent the direction of the current along the $y$-axis and the red arrows represent it along the $x$-axis.)

The surface current distributions on the feedlines and on the cross-shaped components of the CLL and EAD elements are shown in Fig. 5 with only port 1 excited. For convenience, their directions are highlighted with the purple and red arrows. On the one hand, all of the driven and NFRP elements whose currents are oriented along the y-axis (purple arrows) radiate corresponding LP fields into the $+z$-direction (as will be illustrated in detail below). On the other hand, the surface currents along the $x$-axis on all of the driven and NFRP elements contribute to the port isolation performance. First, there are strong surface current distributed on the driven elements, the CLL NFRP element, and the upper surface of EAD NFRP element. As the red arrows highlight, these currents are always oppositely oriented over the entire period. This feature means that the fields radiated by them mutually cancel each other. Therefore, the currents along the $x$-axis do not act as effective radiators when port 1 is excited. Second, the current on the lower surface of the EAD NFRP element along the $x$-axis is too weak to have any noticeable effect on the isolation performance. In the same manner, when port 2 is excited, dual current behaviors are obtained and port 1 is isolated from port 2. Furthermore, these current behaviors must be consistent across the entire $-10-\mathrm{dB}$ bandwidth. With the more than $30 \mathrm{~dB}$ isolation between the two ports achieved by the system over its entire operational bandwidth, these results provide a consistent description of the dual-LP operation.

\section{B. Discussion of the Antenna Loss}

It is noted that the peak realized gain value of our Huygens dipole ESA in either LP state is lower than the theoretical directivity value $4.77 \mathrm{dBi}[18]$. To understand the impact of the dielectric and conductor losses, we simulated the antenna performance by removing the various losses in the same port 1 -only excitation case. First, when simply removing the two baluns and using shorter coaxial cables $(\mathrm{L} 1=5.0 \mathrm{~mm})$, the simulated realized gain (RE) was $2.39 \mathrm{dBi}(62.7 \%)$, indicating that only a $0.19 \mathrm{dBi}(1.4 \%)$ improvement occurs when the baluns are absent. Second, by setting the $\tan \delta=0$ in all five of the substrates, the realized gain and RE values show a significant improvement (1.03 dBi and 19.2\%). Third, when the copper elements were made lossless, the realized gain and RE value improved only a small amount $(0.38 \mathrm{dBi}$ and $6.3 \%)$. Clearly, the dielectric losses associated with all five substrates play the most import role in decreasing the realized gain and $\mathrm{RE}$ values. These results are summarized in Table III.

TABLE III

IMPACT OF THE MATERIAL LOSS PROPERTIES ON THE REALIZED GAIN AND THE RADIATION EFFICIENCY OF THE DUAL-LP HUYGENS DiPOLE ANTENNA

\begin{tabular}{|c|c|c|}
\hline NFRP elements & $\begin{array}{c}\tan \delta=0 \\
\text { (substrate) }\end{array}$ & PEC \\
\hline Only changing the electric elements & $\begin{array}{l}2.67 \mathrm{dBi} \\
(67.4 \%)\end{array}$ & $\begin{array}{c}2.51 \mathrm{dBi} \\
(64.4 \%)\end{array}$ \\
\hline Only changing the magnetic elements & $2.98 \mathrm{dBi}$ & $2.58 \mathrm{dBi}$ \\
& $(73.5 \%)$ & $(64.8 \%)$ \\
\hline Changing both elements & $3.42 \mathrm{dBi}$ & $2.77 \mathrm{dBi}$ \\
& $(81.9 \%)$ & $(69.0 \%)$ \\
\hline No changes & \multicolumn{2}{|c|}{$2.39 \mathrm{dBi}(62.7 \%)$} \\
\hline
\end{tabular}

\section{Explanation of the Directional Radiation}

In order to clarify the broadside radiation mechanism of the dual-LP Huygens dipole ESA, a second version with slightly different design parameters was simulated and optimized to have it radiate in the opposite direction, i.e., along the $-z$-axis. The corresponding optimized design parameters are listed in Table IV. The simulation results are shown in Fig. 6.

When port 1 (port 2) is excited, the resonance frequency is $1.513 \mathrm{GHz}(1.515 \mathrm{GHz})$ with $\left|\mathrm{S}_{11}\right|_{\min }=-17.0 \mathrm{~dB}\left(\left|\mathrm{~S}_{22}\right|_{\min }=-15.8\right.$ 
$\mathrm{dB})$. The corresponding impedance bandwidth is $6.5 \mathrm{MHz}$. The isolation between the two ports is better than $31 \mathrm{~dB}$ within this operational bandwidth. Thus, the total height and electrical size at the resonance frequency $\left(\lambda_{0}=198.28 \mathrm{~mm}\right)$ were $0.0482 \lambda_{0}$ and $k a=0.903$, respectively. The antenna exhibits good backward radiation with a $1.6 \mathrm{dBi}$ peak realized gain along the $-z$-axis, and a $\sim 10 \mathrm{~dB}$ FTBR value. Its radiation efficiency is $60.1 \%$.

TABLE IV

THE OPTIMIZED ESA PARAMETERS (IN MM) OF THE DUAL-LP HUYGENS DIPOLE ANTENNA THAT RADIATES INTO THE -Z-DIRECTION

\begin{tabular}{|c|c|c|c|c|}
\hline$h 1=0.254$ & $h 3=1.508$ & $h 5=0.508$ & $h 6=7.3$ & $h 7=5.2$ \\
\hline$L 2=39.6$ & $L 3=13.3$ & $L 4=13.9$ & $L 5=7.0$ & $L 6=3.1$ \\
\hline$L 7=5.3$ & $L 8=1.54$ & $L 9=0.57$ & $L 10=3.37$ & $W 1=3.1$ \\
\hline$W 2=1.57$ & $W 3=1.0$ & $g 1=1.4$ & $g 2=11.0$ & $g 3=5.0$ \\
\hline$g 4=6.1$ & $g 5=4.0$ & $R 1=28.5$ & $R 2=1.25$ & $R 3=23.4$ \\
\hline$R 4=21.2$ & $R 5=1.8$ & $R 6=2.8$ & $R 7=0.5$ & $R 8=0.6$ \\
\hline$R 9=0.8$ & $\theta=66.8^{\circ}$ & \multicolumn{3}{|c|}{ Null } \\
\hline
\end{tabular}

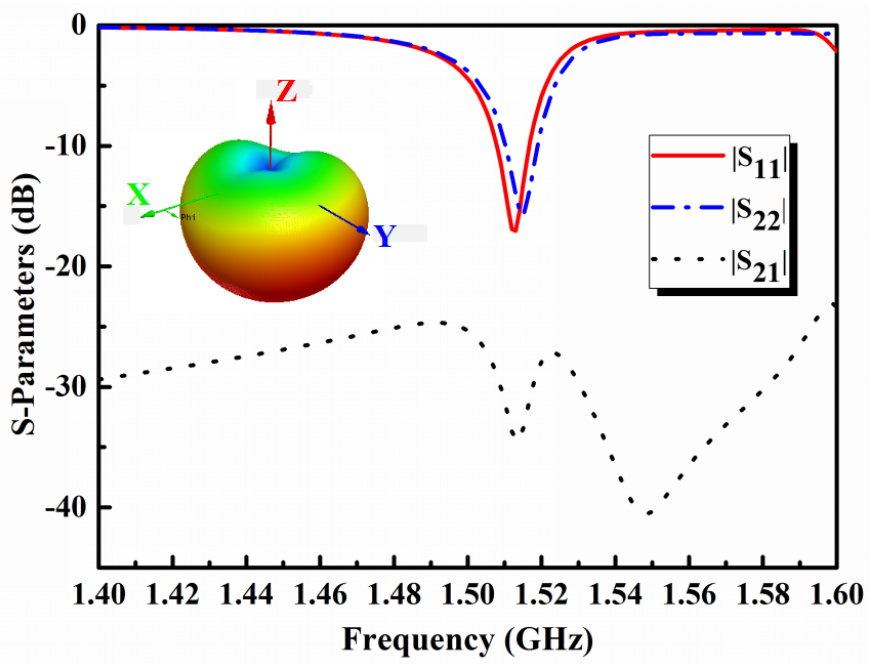

Fig. 6 Simulated S-parameters of the backward radiating dual-LP Huygens dipole ESA, together with its 3-D directivity pattern.

The surface current distributions on the main copper surfaces (i.e., the feedlines and NFRP elements) of this dual-LP Huygens dipole ESA version were simulated, again with only port 1 excited. They are shown in Fig. 7. In comparison with Fig. 5, they are the same along the driven strips, the CLL strips, and the upper surfaces of the electric elements. Consequently, their polarization behaviors are the same. However, in contrast, the currents on the lower surface of the electric elements are not the same. Instead, they have an opposite phase behavior. This means the phases of the "electric dipole" currents witness a $\pi$ phase shift. Therefore, the combined "magnetic dipole" and "electric dipole" elements in this case produce the 3-D cardioid directivity pattern along the - z-axis shown in Fig. 6 .
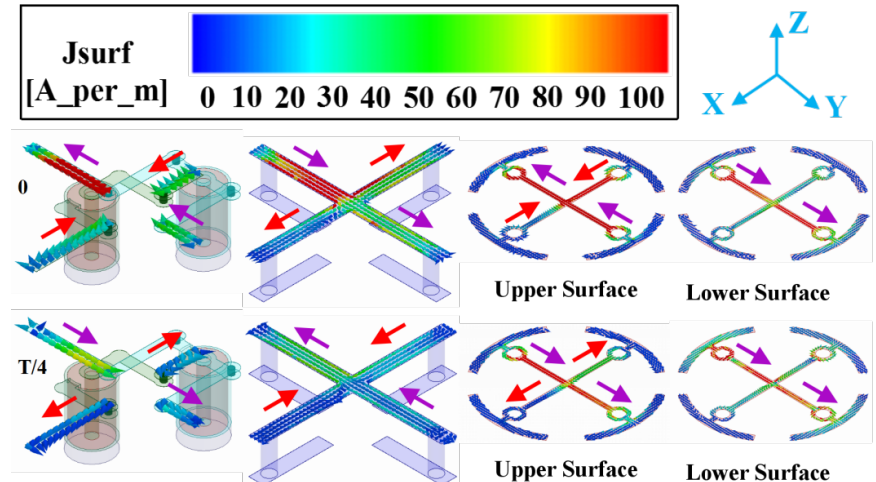

Upper Surface

Lower Surface
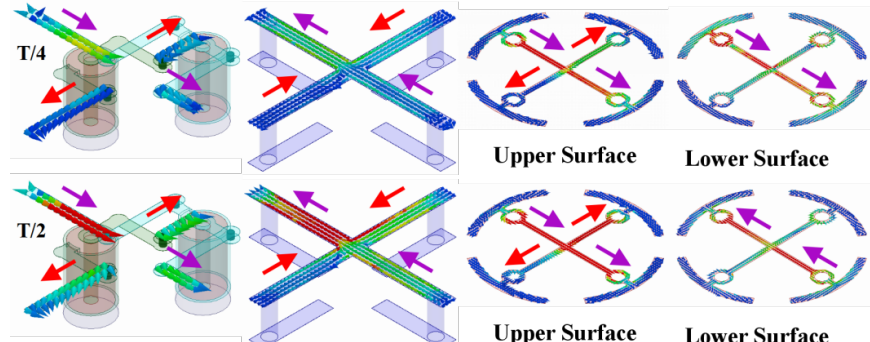

Upper Surface Lower Surface
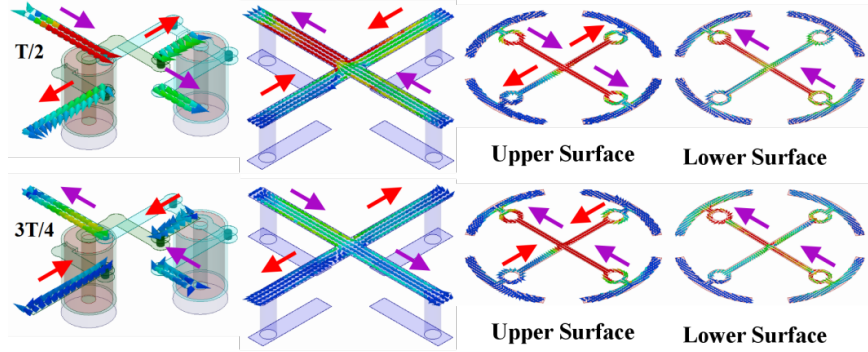

Upper Surface Lower Surface

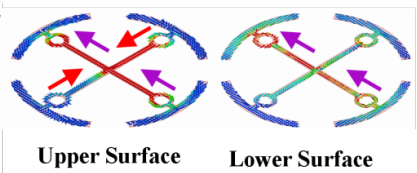

Fig. 7 Surface current distributions on the cross-shaped copper strips of the CLL and EAD NFRP elements and the feedlines of the dual-LP Huygens dipole ESA with backward radiation performance. (The purple arrows represent the direction of the current along the $y$-axis and the red arrows represent it along the $x$-axis.)

\section{CONCLUSION}

In this paper, a dual-LP Huygens dipole ESA was reported. It has a low profile, wide beamwidths, and high FTBR values for both LP states. Moreover, it exhibits high isolation between its two ports. Its five substrate layer design is composed of two pairs of orthogonal CLL and EAD NFRP elements. Both pairs are driven by a corresponding set of orthogonally-oriented dipole strips excited from two ports. Whichever port is active, the LP fields generated by the antenna correspond to the active driven element direction and have a large isolation from the inactive port direction. A prototype dual-LP Huygens dipole ESA was fabricated, assembled and tested. Good agreement between the simulated and measured performance characteristics verifies the effectiveness of the reported design. The operational mechanisms leading to its attractive properties were discussed in detail.

It is anticipated that this dual-LP Huygens dipole ESA would be a very good candidate for future wireless applications needing a high performance, multi-functional, compact, narrow-band antenna system. While a corresponding CP design has been achieved, experimental verification was beyond the scope of the present work. The requisite eight layer structure awaits our access to better and affordable fabrication and assembly technologies.

\section{REFERENCES}

[1]R. F. Harrington, "Effect of antenna size on gain, bandwidth and efficiency," J. Res. Nat. Bur. Stand, vol. 64-D, pp. 1-12, Jan. / Feb. 1960.

[2] Y. Yamada, W. G. Hong, W. H. Jung, and N. Michishita, "High gain design of a very small normal mode helical antenna for RFID tags," in Proc. 
IEEE TENCON, Taipei, Taiwan, Oct./Nov. 2007, pp. 1-4.

[3]W. Hong, Y. Yamada, and N. Michishita, "Low profile small normal mode helical antenna achieving long communication distance," in Proc. Int. Workshop Antenna Technol. (iWAT), Chiba, Japan, Mar. 2008, pp. 167-170.

[4]J. Zhang and Y. Long, "A novel metal-mountable electrically small antenna for RFID tag applications with practical guidelines for the antenna design," IEEE Trans. Antennas Propag., vol. 62, no. 11, pp. 5820-5829, Nov. 2014.

[5]P. Hajizadeh, H. R. Hassani, and S. H. Sedighy, "Planar artificial transmission lines loading for miniaturization of RFID printed quasi-Yagi antenna," IEEE Antennas Wireless Propag. Lett., vol. 12, pp. 464-467, 2013.

[6]A. S. A. Jalal, A. Ismail, A. R. H. Alhawari, M. F. A. Rasid,N. K. Noordin, and M. A. Mahdi, "Metal mount fractal RFID tag antenna with complementary split ring resonator," Prog. Electromagn. Res. C, vol. 39, pp. 25-36, Apr. 2013.

[7]T. Ungan, M. Freunek, M. Muller, W. D. Walker, and L. M. Reindl, "Wireless energy transmission using electrically small antennas," in Proc. IEEE Radio Wireless Symp., San Diego, CA, USA, pp. 526-529, Jan. 2009.

[8]I.-J. Yoon and H. Ling, "Realizing efficient wireless power transfer using small folded cylindrical helix dipoles," IEEE Antennas Wireless Propag. Lett., vol. 9, pp. 846-849, 2010.

[9]D. S. Ricketts, M. J. Chabalko, and A. Hillenius, "Experimental demonstration of the equivalence of inductive and strongly coupled magnetic resonance wireless power transfer," Appl. Phys. Lett., vol. 102, no. 5, p. 053904, Feb. 2013.

[10] Z. Popović, E. A. Falkenstein, D. Costinett, and R. Zane, "Low-power far-field wireless powering for wireless sensors," Proc. IEEE, vol. 101,no. 6, pp. 1397-1409, Jun. 2013.

[11] J. Ng, and R. W. Ziolkowski, "Combining metamaterial-inspired electrically small antennas with electromagnetic band gap (EBG) structures to achieve higher directivities and bandwidths," Proc. 2012 International Workshop on Antenna Technology (iWAT 2012), Tucson, America, pp. 189-192, Mar. 2012.

[12] M.-C. Tang, R. W. Ziolkowski, "Efficient, high directivity, large front-to-back-ratio, electrically small, near-field-resonant-parasitic antenna," IEEE Access, vol. 1, no. 1, pp. 16 - 28, May 2013.

[13] A. D. Yaghjian, T. H. O'Donnell, E. E. Altshuler, and S. R. Best, "Electrically small supergain end-fire arrays," Radio Sci., vol.43, no.RS3002, pp. 1-13, 2008.

[14] A. Noguchi, and H. Arai, "3-element super-directive end-fire array with decoupling network," in Proc.2014 International Symposium on Antennas and Propagation (ISAP), Kaohsiung, Taiwan, Dec. 2-5, 2014.

[15] T. Niemi, P. Alitalo, A. O. Karilainen, and S.A. Tretyakov, "Electrically small Huygens source antenna for linear polarization," IET Microw. Antennas Propag., vol. 6, Iss. 7, pp. 735-739, 2012.

[16] R. W. Ziolkowski, P. Jin, and C.-C. Lin, "Metamaterial-inspired engineering of antennas," Proc. IEEE, vol. 99, no. 10, pp. 1720-1731, Oct. 2011.

[17] R. W. Ziolkowski, "Low profile, broadside radiating, electrically small Huygens source antennas," IEEE Access, vol. 3, pp. 2644-2651, Dec. 2015

[18] M.-C. Tang, H. Wang, and R. W. Ziolkowski, "Design and testing of simple, electrically small, low-profile, Huygens source antennas with broadside radiation performance," IEEE Trans. Antennas Propag., vol.64, no. 11, pp. 4607-4617, Nov. 2016.

[19] M.-C. Tang, T. Shi, and R. W. Ziolkowski, "Electrically small, broadside radiating Huygens source antenna augmented with internal non-Foster elements to increase its bandwidth," IEEE Antennas Wirel. Propag. Lett., vol.16, pp. 712-715, 2017.

[20] M.-C. Tang, B. Zhou, and R. W. Ziolkowski, "Low-profile, electrically small, Huygens source antenna with pattern-reconfigurability that covers the entire azimuthal plane," IEEE Trans. Antennas Propag., vol.65, no. 3 , pp. 1063-1072, Mar. 2017.

[21] M.-C. Tang, T. Shi, R. W. Ziolkowski, "A study of $28 \mathrm{GHz}$, planar, multi-layered, electrically small, broadside radiating, Huygens source antennas," IEEE Trans. Antennas Propag., Special Issue on "Antennas and Propagation Aspects of 5G Communications" vol. 65, no. 12, pp. 6345-6354, Dec. 2017.

[22] P. Alitalo, A. O. Karilainen, T. Niemi, C. R. Simovski, and S. A.Tretyakov, "Design and realisation of an electrically small Huygens source for circular polarization," IET Microwaves, Antennas \& Propagation, vol. 5, no. 7, pp. 783-789, 2011.
[23] C. Morlaas, B. Souny, and A. Chabory, "Helical-ring antenna for hemispherical radiation in circular polarization," IEEE Trans. Antennas Propag., vol. 63, no. 11, pp. 4693-4701, Nov. 2015.

[24] M. Barba, "A high-isolation, wideband and dual-linear polarization patch antenna," IEEE Trans. Antennas Propag., vol. 56, no. 5, pp. 1472-1476, May. 2008.

[25] S.-C. Gao, L.-W. Li, M.-S. Leong, and T.-S. Yeo, "Dual-polarized slot coupled planar antenna with wide bandwidth," IEEE Trans. Antennas Propag., vol. 51, no. 3, pp. 441-448, Mar. 2003.

[26] T.-W. Chiou and K.-L. Wong, "Broad-band dual-polarized single microstrip patch antenna with high isolation and low cross polarization," IEEE Trans. Antennas Propag., vol. 50, no. 3, pp. 399-401, Mar. 2002.

[27] H. W. Lai and K. M. Luk, "Dual polarized patch antenna fed by meandering probes," IEEE Trans. Antennas Propag., vol. 55, no. 9, pp. 2625-2627, Sep. 2007.

[28] Z. Bao, Z. Nie, and X. Zong, "A novel broadband dual-polarization antenna utilizing strong mutual coupling," IEEE Trans. Antennas Propag., vol. 62, no. 1, pp. 450-454, Jan. 2014.

[29] H. Wong, K. L. Lau, and K. M. Luk, "Design of dual-polarized L-probe patch antenna arrays with high isolation," IEEE Trans. Antennas Propag., vol. 52, no. 1, pp. 45-52, Jan. 2004.

[30] K.-M. Mak, H.-W Lai, and K.-M. Luk, "Wideband dual polarized antenna fed by L- and M-probe," in Proc. Asia-Pac. Microw. Conf., pp. 1058-1060, 2012

[31] C. Y. D. Sim, C. C. Chang, and J. S. Row, "Dual-feed dual-polarized patch antenna with low cross polarization and high isolation," IEEE Trans. Antennas Propag., vol. 57, no. 10, pp. 3405-3409, Oct. 2009.

[32] Y. Li, Z. Zhang, W. Chen, Z. Feng, and M. F. Iskander, "A dualpolarization slot antenna using a compact CPW feeding structure," IEEE Antennas Wirel. Propag. Lett., vol. 9, pp. 191-194, 2010.

[33] Y. Li, and K. -M. Luk, " $60-\mathrm{GHz}$ dual-polarized two-dimensional switch-beam wideband antenna array of aperture-coupled magneto-electric dipoles," IEEE Trans. Antennas Propag., vol. 64, no. 2, pp. 554-563, Feb. 2016.

[34] M. Li and K. -M. Luk, "Wideband magneto electric dipole antennas with dual polarization and circular polarization," IEEE Antennas Propag. Mag., vol. 57, no. 1, pp. 110-119, 2015.

[35] S.A. Saario, J.W. Lu and D.V. Thiel, "Full-wave analysis of choking characteristics of sleeve balun on coaxial cables," Electron. Lett., vol.38, no.7, pp. 304-305, Mar. 2002.

[36] C. Icheln, J. Krogerus, and P.Vainikainen, "Use of balun chokes in small-antenna radiation measurements," IEEE Trans. Antennas Propag., vol.53, no. 2, pp. 498-506, Apr. 2004.

[37] M.-C. Tang, and R. W. Ziolkowski, "A study of low-profile, broadside radiation, efficient, electrically small antennas based on complementary split ring resonators," IEEE Trans. Antennas Propag., vol. 61, no. 9, pp. 4419 - 4430, Sep. 2013.

[38] S.-G. Zhou, Z.-H. Peng, G.-L. Huang, and C.-Y.-D. Sim, "Design of a novel wideband and dual-polarized magnetoelectric dipole antenna," IEEE Trans. Antennas Propag., vol. 65, no. 5, pp. 2645-2649, May 2017.

[39] B. Q. Wu and K.-M. Luk, "A broadband dual-polarized magneto-electric dipole antenna with simple feeds," IEEE Antennas Wireless Propag. Lett., vol. 8, pp. 60-63, 2009.

[40] Q. Xue, S. W. Liao, and J. H. Xu, "A differentially-driven dual-polarized magneto-electric dipole antenna," IEEE Trans. Antennas Propag., vol. 61, no. 1, pp. 425-430, Jan. 2013.

[41] Y. Gou, S. Yang, J. Li, and Z. Nie, "A compact dual-polarized printed dipole antenna with high isolation for wideband base station applications," IEEE Trans. Antennas Propag., vol. 62, no. 8, pp. 4392-4395, Aug. 2014. 


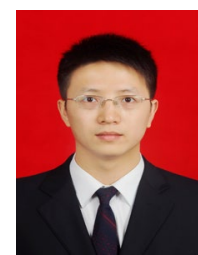

Ming-Chun Tang (S'12-M'13-SM'16) received the B. S. degree in physics from the Neijiang Normal University, Neijiang, China, in 2005 and the $\mathrm{Ph}$. D. degree in radio physics from the University of Electronic Science and Technology of China (UESTC), in 2013. From August 2011 to August 2012, he was also with the Department of Electrical and Computer Engineering, The University of Arizona, Tucson, AZ, USA, as a Visiting Scholar. He is currently a Professor in the College of Communication Engineering, Chongqing University, China. His research interests include electrically small antennas, RF circuits, metamaterial designs and their applications.

Prof. Tang was a recipient of the Best Student Paper Award in the 2010 International Symposium on Signals, Systems and Electronics (ISSSE2010) held in Nanjing, China. He is the founding Chair of the IEEE AP-S / MTT-S Joint Chongqing Chapter. He serves on the Editorial Boards of several journals, including IEEE ACCESS and IET Electronics Letters. He has also served on the review boards of many journals, including the IEEE Transactions on Antennas and Propagation, IEEE Transactions on Microwave Theory and Techniques, IEEE Antennas and Wireless Propagation Letters, IEEE Antennas and Propagation Magazine, IEEE Microwave and Wireless Components Letters, and many international conferences as a General Chair, TPC Member, Session Organizer, and the Session Chair.

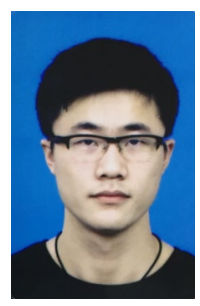

Zhentian Wu was born in China, in 1994. He received the B.S. degree from the West Anhui University, Liuan, China, in 2016. He is currently pursuing the M.S. degree in electronics and communication engineering in the College of Communication Engineering, Chongqing University, China.

He current research interests include electrically small antenna and directional antenna and their applications.

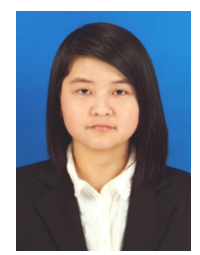

Ting Shi (S'16) received the B.S. degree from the University of Electronic Science and Technology of China (UESTC), Chengdu, China, in 2014 and the M.S. degree from the Chongqing University, Chongqing, China, in 2017. She is currently pursuing the $\mathrm{Ph}$. $\mathrm{D}$ degree in electronics and communication engineering in the College of Communication Engineering, Chongqing University, China.

Her current research interests include non-Foster circuits in electrically small antennas, active antenna applications.

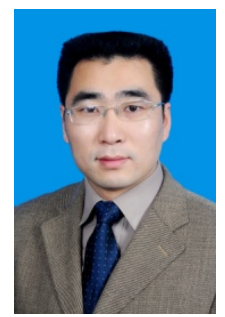

Hao Zeng was born in China, in 1977. He received the M.E and Ph. D degrees in circuit and system from Chongqing University, P. R. China, in 2002 and 2006 respectively.

$\mathrm{He}$ is a Professor in School of communication engineering of Chongqing University China now. From 2010 to 2013, he was a post doctor at University of Electronic Science and Technology, China. Additionally, he worked at Rice University as visiting scholar in USA from 2009 to 2010. His research interests include adaptive array antenna, software defined radio and broadband wireless communication.

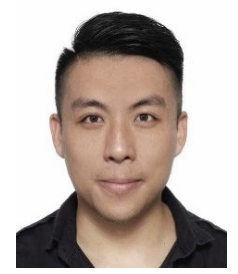

Wei LIN received his $\mathrm{PhD}$ degree in Electronic Engineering from City University of Hong Kong, Hong Kong SAR in August 2016. He received the Master and Bachelor degrees, both in Electronic Engineering, at the South China University of Technology, Guangzhou, China in July 2012 and July 2009, respectively. He worked as a Research Associate at the Nanyang Technological University, Singapore from August 2012 to August 2013.

Dr. Lin is currently a postdoc research fellow with the Global Big Data Technologies Centre, University of Technology Sydney, Ultimo NSW, Australia. Dr. Lin received the Young Scientist Award at the IEEE Region 10 conference (TENCON2015) in December 2015 and a Talent Development Scholarship from the Hong Kong Government in July 2016. He received the Best Poster Paper Award in the 2nd international conference on Electromagnetic Materials and Technologies for the Future (EM-MTF2017) in November 2017 and the Best Young Professional Paper Award (First prize) in the 3rd Australian Microwave Symposium (AMS2018) in February 2018. Dr. Lin was the recipient of the 2018 UTS Early Career Researcher Grant Award from University of Technology Sydney. His research interests include the designs of reconfigurable antennas, HF antennas, satellite antennas, millimeter wave antennas, wireless power transfer, terahertz devices, and their applications.

Dr. Lin has published more than 40 papers in the most prestigious journals and international conferences, which include the IEEE Transactions on Antenna and Propagation; IEEE Transactions on Biomedical Circuits and System; IEEE Access; IEEE Antennas and Wireless Propagation Letters. He serves as reviewers for many IEEE and IET journals.

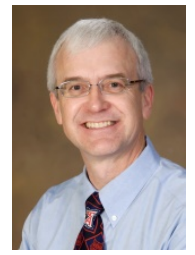

Richard W. Ziolkowski (M'87-SM'91-F'94) received the B.Sc. (magna cum laude) degree (Hons.) in physics from Brown University, Providence, RI, USA, in 1974; the M.S. and $\mathrm{Ph} . \mathrm{D}$. degrees in physics from the University of Illinois at Urbana-Champaign, Urbana, IL, USA, in 1975 and 1980, respectively; and the Honorary Doctorate degree from the Technical University of Denmark, Kongens Lyngby, Denmark in 2012.

He is currently a Distinguished Professor with the University of Technology Sydney, Global Big Data Technologies Centre, Ultimo NSW, Australia. He is also a Litton Industries John M. Leonis Distinguished Professor with the Department of Electrical and Computer Engineering and a Professor with the College of Optical Sciences at The University of Arizona. He was the Computational Electronics and Electromagnetics Thrust Area Leader with the Lawrence Livermore National Laboratory, Engineering Research Division, before joining The University of Arizona, Tucson, AZ, USA, in 1990. He was the Australian DSTO Fulbright Distinguished Chair in Advanced Science and Technology from 2014-2015. He was a 2014 Thomas-Reuters Highly Cited Researcher. His current research interests include the application of new mathematical and numerical methods to linear and nonlinear problems dealing with the interaction of electromagnetic and acoustic waves with complex linear and nonlinear media, as well as metamaterials, metamaterial-inspired structures, and applications-specific configurations.

Prof. Ziolkowski is a Fellow of the Optical Society of America (OSA, 2006), and of the American Physical Society (APS, 2016). He served as the President of the IEEE Antennas and Propagation Society in 2005. He is also actively involved with the URSI, OSA and SPIE professional societies. 